An. MED. InTERNA (Madrid) Vol. 19, N. $^{\circ} 4$, pp. $179-182,2002$

\title{
Trastornos gastrointestinales en Guatemala y su relación con infecciones parasitarias
}

\author{
L. BUJANDA, Mª A. GUTIÉRREZ-STAMPA, C. H. CABALLEROS*, M. E. \\ ALKIZA** \\ Servicio de Medicina Interna. Fundación Barceló. *Hospital Nacional Juan de Dios \\ Rodas. Solola. Guatemala. ** Departamento de Salud Pública. Gobierno Vasco
}

\author{
GASTROINTESTINAL DISORDERS IN GUATEMALA AND THEIR \\ RELATION TO PARASITE INFECTIONS
}

\section{RESUMEN}

Objetivos: Determinar la frecuencia de clínica compatible con trastornos funcionales gastrointestinales en un país subdesarrollado y su relación con infecciones parasitarias.

Material y métodos: Se estudiaron de forma transversal 174 pacientes consecutivos atendidos en una consulta de digestivo en el Hospital de Solola (Guatemala).

Resultados: De todos los pacientes 37 (21\%) presentaban clínica sugestiva de síndrome de intestino irritable, 26 (15\%) de síndrome de intestino irritable más síndrome dispéptico y en 24 (14\%) de síndrome dispéptico. Fueron diagnosticados anteriormente de infección parasitaria gastrointestinal 42 pacientes (24\%). El riesgo relativo de presentar una clínica funcional en estos últimos pacientes era de un OR de 6,33 frente a los que no habían sido diagnosticados de infecciones parasitarias en el pasado. En 59 pacientes (34\%) se realizó determinación de parásitos en heces, de ellos 13 fueron positivos. De todos los pacientes etiquetados clínicamente de trastornos funcionales gastrointestinales 41 pacientes (49\%) habían tenido o tenían alguna infección parasitaria.

Conclusiones: La clínica sugestiva de trastorno funcional gastrointestinal es frecuente en países subdesarrollados. Los pacientes con infecciones parasitarias tienen un mayor riesgo de presentar esta clínica.

PALABRAS CLAVE: Trastornos funcionales. Gastrointestinal. Países subdesarrollados. Infecciones.

\begin{abstract}
Background: To determine the frequency of symptoms sugestive of functional gastrointestinal disorders and their relation to parasite infections in a developing nation.

Material and methods: 174 consecutive patients was seen in a gastroenterology clinic in Solola (Guatemala).

Results: Symptoms sugestive of irritable bowel syndrome was diagnosed in 37 patients $(21 \%)$, irritable bowel syndrome with dyspeptic syndrome in 26 (15\%) and dyspeptic syndrome in 24 (14\%). Forty-two patients $(24 \%)$ had been diagnosed of parasitation in the past. Such patients had a higher risk of suffering symptoms sugestives with functional gastrointestinal disorder (OR 6,335 CI 95\% 2,72-14-75). Fifty-nine patients (34\%) were subjected to parasite examination in stools; of these, 13 proved positive. In this same group of patients the risk of functional gastrointestinal problems was likewise higher among the subjects with parasite infection $(p<0.05)$. In turn, 41 patients $(49 \%)$ with symptoms sugestives of gastrointestinal functional disorders were or had previously been diagnosed of parasite infection.

Conclusions: Symptoms sugestives of functional gastrointestinal disorders are frequent in developing regions and its was in relation to intestinal parasites.
\end{abstract}

KEY WORDS: Functional disorders. Gastrointestinal. Developing countries. Infections.

Bujanda L, Gutiérrez-Stampa $M^{a}$ A, Caballeros $C H$, Alkiza ME. Trastornos gastrointestinales en Guatemala y su relación con infecciones parasitarias. An Med Interna (Madrid) 2002; 19: 179-182.

\section{INTRODUCCIÓN}

Los trastornos funcionales gastrointestinales son muy frecuentes. De ellos un $70 \%$ de pacientes no buscan atención médica $(1,2)$. A pesar de ello, uno de cada 4 pacientes que acude a las consultas médicas, y un $40 \%$ de los pacientes que acuden al médico especialista en gastroenterología, son diagnosticados de trastornos funcionales (3). La prevalencia en países industrializados es del 14 al 24\% de mujeres y del 5 al
$19 \%$ en hombres. La prevalencia es igual en blancos que negros. El gasto sanitario en exploraciones, consultas médicas y tratamientos es muy grande, más aún si se tiene en cuenta que un $50 \%$ de los pacientes toman medicación para sus síntomas (4).

Los estudios de prevalencia de trastornos funcionales en países subdesarrollados son escasos, en algunos la prevalencia es similar a países industrializados $(5,6)$ mientras que en otros es claramente inferior (7). Por otro lado, la etiología de los

Trabajo aceptado: 7 de noviembre de 2001

Correspondencia: Luis Bujanda. C/ Ocharán Mazas 13 Q-1 A. 39700 Castro Urdiales. Cantabria. 
trastornos funcionales digestivos es desconocida, algunos autores han involucrado a las infecciones parasitarias en el desarrollo de estos trastornos.

Nuestro objetivo era conocer la prevalencia de clínica sugestiva de trastorno funcional gastrointestinal en un país subdesarrollado y su relación con las infecciones parasitarias.

\section{MATERIAL Y MÉTODOS}

Se estudió de forma prospectiva, la prevalencia de clínica sugestiva de trastorno funcional en pacientes que acudían de forma consecutiva a consultas de gastroenterología en el Hospital "Juan de Dios de Rodas" de Solola (Guatemala). Este hospital constituye un centro sanitario secundario para una población aproximada de 100.000 habitantes. En esta zona el $80 \%$ de la población es indígena, un $70 \%$ analfabeta y la primera causa de mortalidad es la materno-infantil.

Se analizaron todas las consultas realizadas durante un mes. En total acudieron a esta consulta 174 pacientes. En ellos se analizó la edad, el tipo de síntomas, el tiempo de aparición de la clínica, la presencia de alteración en la deposiciones y si habían sido diagnosticados previamente de infecciones parasitarias por examen de heces. Los síntomas fueron agrupados en patologías según los síntomas en: síndrome dispéptico (SD), síndrome de intestino irritable (SII), reflujo gastroesofágico, úlcus péptico, gastropatía por antiinflamatorios no eseroideos (AINES), estreñimiento, gastroenteritis aguda, patología hepática, patología bilio-pancreática, patología ginecológica, patología osteomuscular y otras.

Se realizó un examen de parásitos en heces cuando se sospechaba que la clínica podía deberse a una infección parasitaria. No fueron realizadas otras pruebas complementarias a no ser que la clínica indicará una patología urgente o enfermedad grave debido a su alto coste económico, a la escasez de recursos materiales y a la dificultad de volver a las consultas. Entre las pruebas complementarias estaban analítica básica (incluía glucosa, creatinina y ALT), hemograma, y excepcionalmente, ecografía abdominal.

Fueron definidos los diferentes grupos de patología según los siguientes criterios clínicos:

El grupo ulcus se definió cuando el paciente refería dolor en epigastrio relacionado con las comidas y/o aliviaba con antiácidos.

El grupo SD era considerado cuando los pacientes presentaban dolor/molestia abdominal localizado en el abdomen superior con una duración mínima de tres meses y síntomas al menos el 25\% del tiempo (por ejemplo; 21 días o más) sin características ulcerosas. En este caso no existían antecedentes de cirugía gástrica, intestinal, biliar o pancreática ni historia previa de enfermedad ulcerosa

El grupo con SII se definió a aquellos pacientes que presentaban una combinación de síntomas gastrointestinales crónicos y recurrentes, atribuídos al intestino y asociado con síntomas de dolor abdominal, alteración del ritmo intestinal y/o distensión abdominal según los criterios de Roma (8).

Se considero el grupo con SD más SII cuando en los pacientes había criterios clínicos de ambas patologías.

Los pacientes con reflujo gastroesofágico eran considerados cuando presentaban como principal síntoma la pirosis independiente de su severidad y frecuencia.
El grupo con gastropatía por AINES se definió a aquellos pacientes con clínica ulcerosa, dispéptica o de reflujo en los que existia el antecedente de consumo de AINES.

Los pacientes con estreñimiento fueron clasificados cuando los pacientes presentaban menos de 3 deposiciones a la semana de más de un año de evolución sin ningún signo o síntoma de alarma.

El grupo con gastroenteritis aguda era definido cuando los pacientes presentaban diarrea con o sin vómitos de menos de 4 semanas de evolución. La diarrea era consideraba cuando había un cambio en el ritmo intestinal con más de 3 deposiciones diarias y una disminución de la consistencia.

Patología osteomuscular era considerada cuando el dolor era claramente relacionado con cambios posturales o movimientos, con dolor muy selectivo a la palpación y no tenía relación con la comida o la deposición.

Patología ginecológica era considerada cuando se relacionaba con la menstruación, el embarazo o existían masas pélvicas que posteriormente eran confirmadas por ecografía.

Se clasificó a los pacientes en otras cuando no se podían englobar en ninguna de las categorías anteriores como por ejemplo, segundas opiniones, clínica atípica, etc.

Se consideraron los pacientes con trastornos funcionales gastrointestinales a los pacientes incluidos en el grupo SD y/o SII.

\section{ANÁLISIS ESTADÍSTICOS}

La comparación entre grupos se realizó mediante la t de Student o el test de Mann Whitney para variables cuantitativas y la prueba de $\chi^{2}$ o prueba exacta de Fisher para variables cualitativas. Una $\mathrm{p}$ igual o menor a 0,05 era considerado como significativa.

\section{RESULTADOS}

De los 174 pacientes, 76 eran hombres y 98 mujeres (H/M; 0,77). La edad media era de 37 años. El tiempo medio de la presencia de síntomas fue de 25 meses.

La clínica que más frecuente presentaron los pacientes fue compatible con trastornos funcionales gastrointestinales (Tabla I). La prevalencia de SII fue superior en mujeres, con una relación hombre/mujer de 0,42 , mientras que el SD fue en hombres con una relación hombre/mujer de 1,42 ( $\mathrm{p}<0,05)$. No hubo diferencias respecto a la edad media en cuanto al tipo de patología que presentaban los pacientes.

En 74 pacientes se realizó alguna prueba complementaria. Hemograma y analítica básica en 18 pacientes (solo uno alterado), parásitos en heces en 59 pacientes, ecografía abdominal en 9 ( 2 patológicas de causa ginecológica) y gastroscopia en 12 (Tabla II).

En 42 (24\%) pacientes se había diagnosticado de infección parasitaria en el pasado (Tabla III). Los pacientes con historia previa de infección parasitaria tenían mayor riesgo de presentar clínica compatible con un trastorno funcional gastrointestinal $(\mathrm{p}<0,05)$, sin observar diferencias según el tipo de parásito intestinal. Se realizó examen de parásitos en heces ante la sospecha de infección en 59 pacientes, de ellos, 13 fueron positivos (Tabla IV). También en este grupo de pacientes se observó como el riesgo de presentar una clínica compatible con un tras- 
torno funcional gastrointestinal era mayor en los pacientes con infección parasitaria $(\mathrm{p}<0,05)$. En total a $41(49 \%)$ pacientes etiquetados de trastorno funcional se había objetivado actualmente o en el pasado de infecciones por parásitos intestinales.

\section{TABLA I}

TIPO DE PATO LOGÍA DIGESTIVA QUE PRESENTABAN LOS PACIENTES SEGÚN LA HISTO RIA CLÍNICA

\begin{tabular}{lc}
\hline Patología & № casos (\%) \\
\hline Síndrome de intestino irritable & $37(21 \%)$ \\
Síndrome de intestino irritable + Síndrome dispéptico & $26(15 \%)$ \\
Síndrome dispéptico & $24(14 \%)$ \\
Ulcus & $20(11 \%)$ \\
Reflujo gastroesofágico & $13(7 \%)$ \\
Otras & $13(7 \%)$ \\
Gastroenteritis aguda & $11(6 \%)$ \\
Osteomuscular & $10(6 \%)$ \\
Gastropatía por AINES & $8(5 \%)$ \\
Ginecológica & $8(5 \%)$ \\
Estreñimiento & $3(2 \%)$ \\
Bilio-pancreática & $1(1 \%)$ \\
Hepática & 0 \\
TOTAL & 174 \\
\hline
\end{tabular}

AINEs: antiinflamatorios no esteroideos.

TABLA II

\begin{tabular}{lcc}
\multicolumn{3}{c}{ TABLA II } \\
\multicolumn{4}{c}{$\begin{array}{c}\text { HALLAZGOS ENDOSCÓ PICO S SEGÚ N } \\
\text { EL GRUPO DE PATO LO GÍA }\end{array}$} \\
\hline Patología & Normal & Anormal \\
\hline SII + SD & $2(1 \mathrm{HP}+$, otro no hecho) & 2 (Gastritis erosiva TU -, \\
Gastritis folicular TU +) \\
Ulcus & $5(3 \mathrm{HP}+, 2 \mathrm{HP}-)$ & 1 (Gastritis aguda antral TU+) \\
Reflujo gastroesofágico & $1(\mathrm{HP}+)$ & 0 \\
Gastropatía por AINES & 0 & 1 (Gastritis aguda antral TU+) \\
TOTAL & $\mathbf{8}$ & 4 \\
\hline
\end{tabular}

AINES: antiinflamatorios no esteroideos; HP: Helicobacter pylori; TU: test de ureasa; SII: síndrome de intestino irritable; SD: síndrome dispéptico.

TABLA III

\begin{tabular}{|c|c|c|c|c|}
\hline \multicolumn{5}{|c|}{ TABLA III } \\
\hline \multicolumn{5}{|c|}{$\begin{array}{l}\text { ANTECEDENTES DE INFECCIO NES PARASITARIAS } \\
\text { EN PACIENTES CON PATO LO GÍA DIGESTIVA }\end{array}$} \\
\hline Parásitos & SII (37 pacientes) & $\begin{array}{c}\text { SII+SD } \\
\text { (26 pacientes) }\end{array}$ & $\begin{array}{c}\text { SD } \\
(24 \text { casos })\end{array}$ & $\begin{array}{c}\text { Resto } \\
\text { Patologías } \\
\text { (87 casos) }\end{array}$ \\
\hline Amebas & 11 & 7 & 4 & 5 \\
\hline Giardias & 1 & 2 & 2 & \\
\hline $\begin{array}{l}\text { Tenias, trichuras } \\
\text { axcaris }\end{array}$ & S, & 1 & 1 & 2 \\
\hline Mas de una & 2 & 2 & & 1 \\
\hline $\begin{array}{l}\text { Otras } \\
\text { TOTAL }\end{array}$ & $15(41 \%)$ & $12(46 \%)$ & $7(29 \%)$ & $8(9 \%)$ \\
\hline
\end{tabular}

SII: síndrome de intestino irritable SD; síndrome dispéptico.
TABLA IV

TIPO DE PARÁSITO ENCONTRADO EN LAS HECES SEGÚN EL TIPO DE PATOLOGÍA

\begin{tabular}{lcccc}
\hline Parásitos & SII (37 pacientes) & $\begin{array}{c}\text { SII+SD } \\
\text { (26 pacientes) }\end{array}$ & $\begin{array}{c}\text { SD } \\
\text { (24 casos) }\end{array}$ & $\begin{array}{c}\text { Resto } \\
\text { Patologías } \\
\text { (87 casos) }\end{array}$ \\
\hline Negativo & 18 & 11 & 7 & 10 \\
$\begin{array}{l}\text { Amebas } \\
\text { Giardias }\end{array}$ & 1 & 3 & 1 & \\
$\begin{array}{l}\text { Ambas } \\
\begin{array}{l}\text { Tenias, trichuras, } \\
\text { axcaris }\end{array}\end{array}$ & 4 & 1 & & \\
$\begin{array}{l}\text { Otras } \\
\% \text { de positivos }\end{array}$ & $25 \%(6 / 24)$ & $31 \%(5 / 16)$ & $22 \%(2 / 9)$ & $0 \%(0 / 10)$ \\
\hline
\end{tabular}

$\mathrm{SCl}$ : síndrome de intestino irritable; SD: síndrome dispéptico.

\section{DISCUSIÓN}

La prevalencia en los países occidentales de SII y SD es de $15-20 \%(8-10)$ y un $20-40 \%$, respectivamente $(1-3,11)$. Un $40 \%$ de todas las consultas realizadas a los especialistas de gastroenterología son trastornos funcionales. Estudios de prevalencia realizados en países subdesarrolados muestran como China y Nigeria presentan una prevalencia similar a países occidentales $(5,6)$. Sin embargo, otros estudios muestran como la prevalencia en poblaciones rurales, otras zonas subdesarrolladas como el sureste asiático, oriente medio y en hispanos que emigran a USA es inferior a zonas industrializadas (7,12-14). En nuestro estudio se observó como la clínica funcional en una zona subdesarrollada era muy alta, igual o mayor, que en países industrializados $(50 \%)$, y al igual que otros estudios, se observaba un predominio de mujeres en el SII, hecho que no ocurre en otros países subdesarrollados como la India y Sri Lanka. En Guatemala a pesar de ser un país subdesarrollado, el acceso de la mujer a la sanidad era similar a los hombres.

También al igual a lo observado en los países industrializados se observa como existe un solapamiento entre pacientes con SD y SII. Así, entre un $23 \%$ y $50 \%$ de todos pacientes con clínica dispéptica tenían una clínica compatible con SII $(10,15)$ y al revés, entre un 18 y $88 \%$ de los pacientes con SII tienen dispepsia. En nuestro estudio se observó unas proporciones similares $(52 \%$ y $41 \%$, respectivamente) destacando mucho más la presencia de síntomas de SII.

Entre un 15-25\% de pacientes dispépticos tienen causa orgánica. Entre las causas orgánicas más frecuentes están la úlcera péptica, la enfermedad por reflujo gastroesofágico, la pancreatitis crónica, la enfermedad celiaca, la intolerancia a la lactosa, algunos fármacos, las enfermedades infiltrativas del estómago, los trastornos metabólicos, el hepatocarcinoma y la angina intestinal (16). En muy pocas ocasiones se considera las infecciones parasitarias como causa de los trastornos funcionales. En nuestro estudio un $15 \%$ de los pacientes etiquetados de SD y/o SII estaban infectados (27\% de todas las muestras analizadas). Es conocido como ciertos parásitos como la amebiasis o la giardiasis puede simular un cuadro funcional digestivo. En nuestro estudio más de la mitad de los casos con trastornos funcionales digestivos tenían alguno de estos dos parásitos. 
La fisiopatología de los trastornos funcionales gastrointestinales (SII y/o SD) es desconocida (17). Muchas hipótesis han sido propuestas como alteración de la motilidad gastrointestinal, alteración nerviosa, alteraciones hormonales, trastornos psicológicos o psiquiátricos, trastornos en la percepción visceral, factores dietéticos y factores genéticos. Sin embargo, todas estos factores o alteraciones pueden encontrarse en sujetos sanos sin síntomas, tampoco existe una clara relación causa-efecto, y por otro lado, el tratamiento de estas alteraciones no se traduce en una mejoría de los síntomas. Las infecciones, tanto bacterianas como parasitarias, se ha intentado involucrar en la etiopatogenia de los trastornos funcionales digestivos fundamentalmente en el SII (18-20). El mecanismo por el cual producirían los síntomas es desconocido. Se piensa que la infección puede invadir la pared intestinal y afectar a la musculatura e inervación intestinal alterando la motilidad y sensibilidad intestinal $(21,22)$. Otro mecanismo que podría contribuir sería la malabsorción de ácidos biliares que produciría un aumento del peristaltismo intestinal (23).

En resumen, observamos como los síntomas sugestivos de trastornos funcionales digestivos son frecuentes en algunos países subdesarrollados y como una gran parte de estos pacientes estaban o habían estado infectados por parásitos intestinales.

\section{Bibliografía}

1. Friedman LS. Helicobacter pylori and nonulcer dyspepsia. N Engl J Med 1998; 339: 1928-1930.

2. Penston JG, Pounder RE. A survey of dyspepsia in Great Britain. Aliment Pharmacol Ther 1996; 10: 83-89.

3. Fisher RS, Parkman HP. Management of nonulcer dyspepsia. Management of nonulcer dyspepsia. N Engl J Med 1998; 339: 1376-1381.

4. Nyren O, Adami HO, Nyberg A. Social and economic effects of nonulcer dyspepsia. Scand J Gastroenterol 1985; 20 (Supl 109): 41-45.

5. Bi-zhen W, Qi-Ying P. Functional bowel disorders in apparently healthy Chinese people. Chinese J Epid 1988; 9: 345-349.

6. Olubuyide IO, Olawuyi F, Fasanmade AA. A study of irritable bowel syndrome diagnosed by Manning criteria in an African population. Dig Dis Sci 1995; 40: 983-985

7. Danivat D, Tankeyoon M, Sriratanaban A. Prevalence of irritable bowel syndrome in a non-Western population. BMJ 1988; 296: 1710.

8. Drossman DA, Whitehead WE, Camilleri M. Irritable bowel syndrome: a technical for practice guideline development. Gastroenterology 1997; 112: 2120-2137.

9. Drossman DA, Thompson WG. The irritable bowel syndrome: review and a graduated multicomponent treatment approach. Ann Inter Med 1992; 116: 1009-1016.

10. Caballero-Plasencia AM, Sofos-Kontoyannis S, Valenzuela-Barranco M, Martín-Ruiz JL, Casado Caballero FJ, Guilarte López-Mañas J. Irritable bowel syndrome in patients with dyspepsia: a community-based study in southern Europe. Eur J Gastroenterol Hepatol 1999; 11: 517-522.

11. Mearin F. Dispepsia funcional: concepto y epidemiología. Rev Esp Enf Digest 1997; 89: 80-83.

12. Zuckerman MJ, Guerra LG, Drossman DA, Foland JA, Gregory GG. Comparison of bowel patterns in hispanics and non-hispanic whites. Dig Dis Sci 1995; 40: 2647-2655.
13. AGA. Medical position statement: irritable bowel syndrome. Gastroenterology 1997; 112: 2118.

14. Massarrat S, Saberi-Firoozi M, Soleimani A, Himmelmann GW, Hitzges M, Keshavarz H. Peptic ulcer disease, irritable bowel syndrome and constipation in two populations in Iran. Eur J Gastroenterol Hepatol 1995; 7: 427-433.

15. Holtmann G, Goebell H, Talley NJ. Functional dyspepsia and irritable bowel syndrome. Am J Gastroenterol 1997; 92:954-9.

16. Talley NJ, Silverstein MD, Agréus L, Nyrén O, Sonnenberg A, Holtmann G. AGA technical review, evaluation of dyspepsia. Gastroenterology 1998; 114: 579-595.

17. Holtmann G, Goebell H, Talley NJ. Functional dyspepsia and irritable bowel syndrome: is there a common pathophysiological basis?. Am J Gastroenterol 1999; 92: 954-959.

18. Giacometii A, Cirioni O, Fiorentini A, Fortuna M, Scalise G. Irritable bowel syndrome in patients with Blastocystis hominis infection. Eur J Clin Microbiol Infect Dis 1999; 18: 436-439.

19. Anand AC, Reddy PS, Saiprasad GS, Kher SK. Does non-dysenteric intestinal amoebiasis exist? Lancet 1997; 349: 89-92.

20. Neal. KR, Hebden J, Spiller R. Prevalence of gastrointestinal symptoms six months after bacterial gastroenteritis and risk factors for development of the irritable bowel syndrome: postal survey patients. BMJ 1997; 314: 779-782.

21. Collins SM, Barbara G, Vallance B. Stress, inflamation and the irritable bowel syndrome. Can J Gastroenterol 1999; 13 (Suppl A):47A-49A.

22. Barbara G, Vallance BA, Collins SM. Persistent intestinal neuromuscular dysfunction after acute nematode infection in mice. Gastroenterology 1997; 113: 1224-1232.

23. Niaz SK, Sandrasegaran K, Renny FH, Jones BJ. Postinfective diarrhoea and bile malabsorption. J R Coll Physicians Lond 1997; 31: 53-56.

\section{FE DE ERRATAS}

El trabajo "Trastornos gastrointestinales en Guatemala y su relación con infecciones parasitarias" fue publicado en el $\mathrm{n}^{\circ}$ de Enero de 2002 pags. 3-6. Por un error en la impresión fue publicado anómalamente, apareciendo el texto de sus distintos apartados, mezclado de forma inconexa. La versión que aparece en este número, págs. 179-182, es la original y válida. 